\title{
Educación para la adaptación. Actualidad de la "teoría de la pseudocultura" de T. W. Adorno en tiempos del aprendizaje permanente
}

\author{
Education for adaptation. Actuality of the "theory of \\ balf-education" of T. W. Adorno in times of lifelong learning \\ Dr. Manuel Rühle \\ DGB Bildungswerk Bayern \\ manuel.ruehle@bildungswerk-bayern.de
}

DOI: http://doi.org/10.15366/bp2019.21.005

Bajo Palabra. II Época. No21. Pgs: 97-108 


\begin{abstract}
La presente comunicación tiene por objeto los desarrollos actuales en el campo de la educación en el capitalismo global que, sirviéndose de las obras filosófico-sociológicas de Adorno, pueden ser interpretados como una industrialización cultural de la pedagogía. Partiendo de una breve exposición del concepto de pseudocultura (Halbbildung) como forma de la conciencia cultural-industrial, se sostendrá la tesis de que, en el capitalismo post-fordista del presente, una pedagogía cultural-industrial contribuye de manera decisiva a la producción sistemática y la consolidación sostenible de la conciencia 'pseudocultivada'.
\end{abstract}

Palabras Clave: teoría de la educación, economización de la educación, industria cultural, pseudocultura, T. W. Adorno, experiencia.

\section{Resumen}

The article focuses on current trends in the education sector which, making reference to Adorno's works on social and cultural formation philosophy, are interpreted as a progressive cultural industrialization of pedagogy in theory and practice. Departing from a brief definition of the concept of half-education (Halbbildung) as the cultural industrial form of consciousness, the thesis will be exposed, that under the conditions of contemporary post-fordistic capitalism an almost exclusively economically oriented cultural industrial pedagogy contributes decisively to the social production of half-educated consciousness.

Keywords: philosophy of education, economization of education, cultural industry, semi-formation, T. W. Adorno, experience. 
L

as siguientes consideraciones pretenden poner a la vista ciertos desarrollos actuales del campo de la educación en el capitalismo global, sirviéndose de las herramientas de la teoría crítica de T. W. Adorno. En su conferencia de 1959: Teoría de la psendocultura, Adorno reflexiona sobre el declive de la idea burguesa de Bildung --que en esta conferencia denominaré como cultivación-, como consecuencia del fracaso de su realización histórico-social. La cultivación está sometida bajo la misma dialéctica del declive que la cultura burguesa en su totalidad: a la comodificación progresiva de todas las producciones culturales y la consiguiente eliminación de su contenido crítico corresponde la cosificación progresiva de los sujetos receptores. Estas ideas serán retomadas y aplicadas a las relaciones sociales del presente. El foco estará puesto en la pregunta por la forma actual de la pseudocultura como resultado de los procesos de aprendizaje políticamente deseados.

Voy a comenzar con un bosquejo del concepto de la pseudocultura en el contexto de la teoría de la industria cultural de Adorno y Horkheimer (1.). Este bosquejo me servirá como marco analítico para poner a la vista la idea de la educación actualmente dominante como una educación para la permanente adaptación a las exigencias de la acumulación capitalista (2.). Finalmente, voy a hacer referencia al concepto de la experiencia mental que Adorno expone en la Dialéctica negativa con el fin de formular una propuesta para el desarrollo de una pedagogía crítica (3.).

\section{Pseudocultura socializada como forma de conciencia cultural-industrial}

Con la transición de la sociedad burguesa clásica del siglo XIX al 'capitalismo tardío' del siglo XX, adviene una subordinación amplia de objetivaciones culturales de todo tipo bajo los imperativos de producción y consumo capitalistas. Este proceso lleva a una unificación y equiparación estructurales de los bienes culturales por medio de las cuales éstos son integrados en una totalidad más o menos cerrada: la industria cultural. "Hoy la cultura lo hace todo semejante. Cine, radio y revistas constituyen un sistema. Cada sector está armonizado en sí mismo y todos entre ellos" ${ }^{1}$. No es que los productos cultural-industriales sean también

\footnotetext{
${ }^{1}$ Adorno, T. W., Dialéctica de la ilustración. Fragmentos filosóficos [= Obra completa, 3, edición de Rolf Tiedemann, traducción de Joaquín Chamorro Mielke], Madrid, Akal, 2007, p. 133.
} 
mercancías, como era el caso con las obras de arte del siglo XIX, sino que ahora son del todo mercancías. En consecuencia, pierden cualquier capacidad de trascender y son completamente funcionalizados conforme a las necesidades reproductivas de la sociedad. En tanto que mercancías de consumo puras, los productos cultural-industriales deben tener una constitución que los hace accesibles sin esfuerzo alguno y que no exige ninguna actividad propia por parte del receptor. Así, consisten en repeticiones permanentes, estereotipos, clichés y efectos que son puestos en serie de manera esquemática, sin ningún tipo de relación interna. Simultáneamente, son presentados una y otra vez bajo apariencias técnicamente nuevas y son anunciados como acontecimientos sin comparación. Los contenidos específicos de cada producto pierden su importancia, sólo sirven como ocasión para aplicar los esquemas ideológicos. Su mensaje se reduce al llamamiento constante de que la adaptación sin reservas a lo dado es la condición imprescindible para tener éxito social, o bien, para conservar la propia existencia:

El imperativo categórico de la industria cultural no tiene nada que ver con la libertad, a diferencia del imperativo categórico de Kant. Dice así: 'acomódate, aunque no sepas a qué; acomódate a lo que existe y a lo que todos piensan como reflejo del poder y la omnipresencia de lo que existe'. Mediante la ideología de la industria cultural, la adaptación sustituye a la conciencia ${ }^{2}$.

La forma de la conciencia humana resultante del proceso de socialización cultural-industrial es lo que Adorno denomina como pseudocultura (Halbbildung) ${ }^{3}$. Así como la cultura en su lado objetivo se convierte en industria cultural, también la cultivación como "cultura según el aspecto de su apropiación subjetiva" ${ }^{4}$ sucumbe a un proceso de transformación de graves consecuencias. En sus orígenes

\footnotetext{
${ }^{2}$ Adorno, T. W., Crítica de la cultura y socidedad I: Prismas. Sin imagen directriz [= Obra completa, 10/1, edición de Rolf Tiedemann, traducción de Jorge Navarro Pérez], Madrid, Akal, 2008, p. 300.

${ }^{3}$ En el contexto de la teoría crítica, el término 'pseudocultura' [Halbbildung] aparece por primera vez en la Dialéctica de la ilustración de 1944, más exactamente en el apartado VI del capítulo "Elementos del antisemitismo. Límites de la ilustración” (cfr. Adorno, T. W., Dialéctica de la ilustración, op. cit., p. 212 ss.). Adorno y Horkheimer utilizan el término para denotar la forma de la conciencia dominante bajo las condiciones sociales del 'capitalismo tardío', para hacer comprensible por qué el antisemitismo eliminatorio del nacional-socialismo de la Alemania de entonces cayó en un terreno así de fértil. La breve sección de la Dialéctica de la ilustración ya menciona todos los puntos esenciales que Adorno expone quince años después en su conferencia titulada "Teoría de la pseudo-cultura" (cfr. Adorno, T. W., Escritos sociológicos $I$ [ = Obra completa, 8, edición de Rolf Tiedemann, traducción de Agustín González Ruiz], Madrid, Akal, 2004, p. 86 ss.).

A consideración del autor, el término castellano 'pseudocultura' corresponde al concepto de 'Halbbildung' de manera más precisa que la expresión 'media cultura'. En este sentido nos alejamos de la traducción de Joaquín Chamorro Mielke en el volumen 3 de la edición de Akal y seguimos la traducción de Agustín González Ruiz en el volumen 8 de la misma edición.
}

${ }^{4}$ Adorno, T. W., Escritos sociológicos I, op. cit., p. 87. 
modernos en la época de la ilustración, la cultivación puede considerarse como la expresión sistemática del pensamiento humanista burgués. Ella designa, en pocas palabras, una forma de relación consigo y con el mundo que no se caracteriza por la represión de la naturaleza pulsional del ser humano, sino por la integración de sus diversas propiedades esenciales en una totalidad armónica. Sin embargo, como la burguesía no reflexionó sobre las condiciones histórico-sociales de la realización de esta pretensión, sino que entendió la cultivación exclusivamente como cultura del espiritu, el contenido utópico de ésta no sólo se desvaneció, sino que, más bien, se convirtió en lo contrario. Concretamente, esto se realizó bajo la forma de una escisión progresiva de los polos dialécticos del concepto de la cultivación, por una parte, en cuanto que formación fáctica para los fines de la economía capitalista y, por otra parte, en cuanto que idealización contrafáctica de su supuesta liberación de toda finalidad 5 .

En el capitalismo organizado del siglo XX, la cultivación se ha convertido, finalmente, en "pseudocultura socializada, en la omnipresencia del espíritu alienado". Ella se destaca, esencialmente, por haber extraviado toda distancia crítica con respecto a la realidad social. Sólo puede captar aquello que ya ha sido seleccionado y preformado por el esquematismo cultural-industrial. En lugar de un intercambio vivo entre sujeto y objeto, se impone una reacción irreflexiva y abrupta según los estímulos situacionales:

La experiencia, la continuidad de la [conciencia] en la que dura lo no presente, en la que el ejercicio y la asociación fundan la tradición en cada individuo, se ve sustituida por el estar informado puntualmente, sin compromiso de forma cambiable y efímera, respecto de lo que ha de señalarse ya que se verá borrado un instante después por otras informaciones ${ }^{7}$.

Esto crea en el sujeto una carencia fundamental de significado y relación de lo percibido, que no puede situarse en un contexto de sentido abarcador y que hace imposible tanto la apertura de la realidad material y espiritual, como la constitución de una identidad personal coherente. La consecuencia es la cosificación de la relación de lo humano consigo mismo y con el mundo: "La pseudocultura es el espíritu tomado por el carácter fetichista de la mercancía" ${ }^{8}$. En este sentido se puede caracterizar la pseudocultura como una profunda incapacidad de tener experiencias.

\footnotetext{
5 Cfr. ibid., p. 97 s.

${ }^{6}$ Ibid., p. 84.

7 Ibid., p. 108.

${ }^{8}$ Ibid., p. 101.
} 


\section{El aprendizaje permanente como auto-adaptación permanente}

Sobre la aCtUalidad fundamental de la teoría de la industria cultural no puede haber mayores dudas. Por el contrario, con la llamada 'revolución microelectrónica' y la expansión y multiplicación de las influencias cultural-industriales, las tesis de Horkheimer y Adorno ganan una renovada vigencia. Sin embargo, no se seguirán aquí las formas observables del consumo cultural presente, sino que se pondrá a la vista el ámbito donde la dialéctica del declive de la idea de la cultivación se articula, por así decirlo, de forma pura. Esto es, en los discursos dominantes sobre la educación en Europa durante los últimos años. Se defenderá la tesis de que en estos discursos pueden identificarse, en varios sentidos, tendencias progresivas de una culturindustrialización de la pedagogía en teoría y práctica.

Una de las tareas básicas de la actividad estatal en las sociedades capitalistas es garantizar la acumulación de capital sin interferencias. En el capitalismo postfordista del presente, esta exigencia se manifiesta, como dice el politólogo alemán Joachim Hirsch, "en la orientación de todas las esferas de la sociedad hacia el objetivo de la

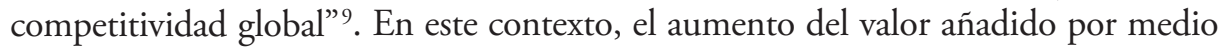
de la calificación intensificada de la fuerza de trabajo humana, juega un papel central. Una expresión característica de estas tendencias es el concepto de las 'calificaciones clave' [Schlüsselqualifikationen], formulado a principios de la década de 1970, en el contexto de una reorganización general de las formas de trabajo y de producción. En palabras de Dieter Mertens, inventor del concepto, este debía asegurar la "capacidad de adaptación a lo no-pronosticable" ${ }^{10}$ por parte de los trabajadores. Su continuación actual se encuentra en los modelos de competencias, que, por ejemplo, sirven de base para las pruebas PISA de la Organización para la Cooperación y el Desarrollo Económico (OCDE). En este marco, se entiende por competencia una aptitud universalmente aplicable para la resolución de problemas cotidianos: "PISA evalúa la capacidad de completar tareas relacionadas con la vida real" ${ }^{11}$. Esta capacidad debe cimentar un proceso de aprendizaje para toda la vida, cuyas magnitudes de referencia consisten en las exigencias sociales correspondientes: "PISA se basa en un modelo dinámico de aprendizaje permanente en el cual los nuevos conocimientos y aptitudes

\footnotetext{
${ }^{9}$ Hirsch, J., Materialistische Staatstheorie. Transformationsprozesse des kapitalistischen Staatensystems, Hamburg, VSA, 2005, p. 150.

${ }^{10}$ Mertens, D., "Schlüsselqualifikationen. Thesen zur Schulung für eine moderne Gesellschaft", en Lange, Elmar y Büschges, Günter (eds.), Aspekte der Berufswahl in der modernen Gesellschaft, Frankfurt a. M., Aspekte-Verlag, 1975 , p. 411.

${ }^{11}$ OCDE, Conocimientos y aptitudes para la vida. Primeros resultados del programa internacional de evaluación de estudiantes (PISA) 2000 de la OCDE, México, Santillana, 2001, p. 20.
} 
necesarias para adaptarse con éxito a un mundo cambiante se obtienen continuamente durante toda la vida" ${ }^{12}$. El enfoque sobre la meta de la adaptación es programático para la idea de la educación dominante y reducida a lo económico que entiende al individuo casi exclusivamente como recurso humano. Aquí, la tarea de los procesos pedagógicos consiste en la producción de las condiciones para la explotación económica de este recurso, es decir, en transformarlo en capital humano ${ }^{13}$. El concepto del 'aprendizaje permanente' representa de cierta manera la variante neoliberal de esta exigencia capitalista básica, en la medida en que esta transformación se debe realizar en tanto que auto-transformación, según la lógica de la auto-optimización continua. El sujeto debe considerarse como un 'proyecto' siempre deficitario que orienta su auto-management según la situación actual del mercado. Según el Memorándum sobre el aprendizaje permanente de la Unión Europea del año 2000, un documento central dentro del discurso, la tarea de cada uno es "aprender a aprender, a adaptarse a los cambios y a gestionar enormes flujos de información" ${ }^{14}$. En la versión española de este documento, la raíz de la palabra 'adapt-' se encuentra, en total, 19 veces en el cuerpo del texto, siempre con una connotación exclusivamente positiva; además, en el anexo se encuentra, como ejemplo 'best-practice', un proyecto del campo de la cualificación laboral que lleva exactamente el mismo nombre: 'ADAPT'.

La conformidad con el mercado de los procesos educativos en el sentido del 'aprendizaje permanente' se debe asegurar con ayuda de un régimen de certificación y evaluación en todos los campos del sistema educativo. Por su parte, este régimen se ve flanqueado por una investigación educativa empírica cuyas medidas se extraen de manera prescriptiva a partir de las expectativas sociales definidas ${ }^{15}$. Al mismo tiempo, se intenta identificar y hacer comparables los conocimientos y capacidades individuales mucho más allá de la medida clásica de los exámenes escolares con el fin de impulsar la co-modificación de la fuerza de trabajo humana, pero también de la educación misma. A parte de las pruebas PISA, puede mencionarse a manera de ejemplo el Marco Europeo de Cualificaciones para el aprendizaje permanente, como instrumento para mejorar la comparabilidad de los programas de formación en Europa ${ }^{16}$.

${ }^{12}$ Ibid., p. 14.

${ }^{13}$ Cfr. Bernhard, A., Biopiraterie in der Bildung. Einsprüche gegen die vorherrschende Bildungspolitik, Hannover, Offizin, 2010.

${ }_{14}$ CCE, Memorándum sobre el aprendizaje permanente, Bruselas, 2000, p. 12. Documento online disponible en: http://www.oei.es/historico/eduytrabajo2/Memoaprenpermanente.pdf (consultado el 10.06.2018).

${ }_{15}$ Cfr. Dammer, K.-H., Vermessene Bildungsforschung. Wissenschaftshistorische Hintergründe zu einem neoliberalen Herrschaftsinstrument, Baltmannsweiler, Schneider Hohengehren, 2015, pp. 107 ss.

${ }^{16} \mathrm{Cfr}$. CE, El Marco Europeo de Cualificaciones para el aprendizaje permanente (EQF-MEC), Luxemburgo, Oficina de Publicaciones Oficiales de las Comunidades Europeas, 2009. 
Estas tendencias se pueden resumir como el intento de una uniformización impuesta de la educación, tanto formal como no-formal, según imperativos económicos. Este intento apunta a la eliminación del elemento crítico-reflexivo de la educación para beneficio de la generación de una disponibilidad subjetiva y una voluntad de adaptación sin reservas. De esta manera, el resultado deseado de estas tendencias corresponde exactamente a los rasgos de la pseudocultura, como han sido expuestos por Adorno: "la educación se reduce a los distintivos de la inmanencia social y del estar integrado y se convierte ella misma sin reservas en algo intercambiable, aprovechable" ${ }^{17}$. Toda posibilidad de tomar una distancia mental respecto de las exigencias y expectativas de la sociedad debe cerrarse. Así, mientras que en los tiempos de Adorno la pseudocultura era primariamente un resultado cultural-industrial, desde entonces ésta devino progresivamente, a la vez, objeto de una política educativa que la exige en el nombre de la competitividad internacional. En este proceso, los sistemas educativos, tanto privados como públicos, se juntaron con las agencias de la cultura de masas, dedicándose a la producción sistemática y la consolidación sostenible de la conciencia 'pseudocultivada'. Así, la pedagogía se pervierte tendencialmente, tanto en sus formas como en sus contenidos, en una pedagogía cultural-industrial, es decir, en una pedagogía cuyo motivo principal es la co-modificación de las potencias humanas.

\section{Perspectivas críticas: la experiencia mental como experiencia formadora}

Si LA PSEUdocultura SE EXPRESA en la neutralización de la capacidad de tener experiencias, entonces el desarrollo de esta capacidad puede ser considerado como rasgo fundamental de una idea crítica de la cultivación. Para la conceptualización de una teoría y práctica emancipatorias de la educación son de gran relevancia las reflexiones de Adorno, especialmente las que expone en la Dialéctica negativa. Mientras que el sentido común --como también el positivismo científico resultante del mismo-, hace equivaler lo particular al esquema general del concepto y lo elimina fácticamente en cuanto tal, el pensamiento dialéctico negativo intenta salvaguardar la particularidad de lo singular en el acto de conocimiento. Describe "el empeño de llegar más allá del concepto por medio del concepto" ${ }^{18}$, es decir,

\footnotetext{
${ }_{17}$ Adorno, T. W., Escritos sociológicos I, op. cit., p. 107.

18 Adorno, T. W., Dialéctica negtiva / La jerga de la autenticidad [=Obra completa, 6, edición de Rolf Tiedemann, traducción de Alfredo Brotons Muñoz], Madrid, Akal, 2005, p. 26.
} 
el intento continuo de corregir la rigidez identificadora del concepto por medio de la obtención de lo que no se puede asir conceptualmente: lo no-idéntico. Esta superación de la limitación esencial del concepto solo se puede realizar de manera aproximada a través de constelaciones de conceptos que se agrupan alrededor de la cosa. En esta dedicación sin reservas a lo no-idéntico consiste el núcleo de la experiencia mental o filosófica como la entiende Adorno:

Tendría su contenido en la diversidad, no aprestada por un esquema de objetos que se le imponen o que ella busca; se abandonaría verdaderamente a ellos, no los utilizaría como espejos en los que reproducirse, confundiendo su copia con la concreción. No sería otra cosa que la experiencia plena, no reducida, en el medio de la reflexión conceptual ${ }^{19}$.

En este sentido, la experiencia puede comprenderse a la vez como condición $y$ elemento del proceso de la cultivación: la modificación formadora del sujeto en cuanto que diferenciación de sus potencias perceptivas y reflexivas se basa esencialmente en la apertura experiencial de la realidad sensorial y mental socialmente mediada. Esta apertura, por su parte, constituye la idea misma de apropiación del mundo en el modo de la cultivación.

Todo intento de explorar perspectivas viables para procesos formadores en el sentido de la experiencia mental exige, en primer lugar, una auto-reflexión radicalmente crítica de los sujetos implicados. Dado que la pseudocultura consiste en el resultado de procesos organizados de aprendizaje, dicha auto-reflexión implica, por parte de los agentes pedagógicos, la necesidad de una aclaración de su concepción de educación frente a las relaciones sociales de dominio. Además de la auto-ilustración de los elementos cultural-industriales de la propia estructura de la personalidad, es preciso, además, crear conciencia sobre la mediación de las propias formas de acción pedagógica para prevenir la asimilación inconsciente de principios cultural-industriales. Por supuesto, los procesos de experiencia mental no pueden ser inducidos deliberadamente, sino, en el mejor de los casos, provocados, al intentar liberar la conciencia cotidiana de sus caminos habituales. Como idea didáctica central, puede servir el factor de la ruptura con la estereotipia cultural-industrial: la apertura de nuevas perspectivas sobre la realidad exige que la validez incuestionada del esquematismo se suspenda, aunque sea temporalmente, se tome en consideración y se problematice. La educación no debe, a la manera de una interpretación ingenua del 'aprendizaje experiencial', llevar a la continuación con otros medios de la experiencia cotidiana de los aprendices, sino que debe posibilitar su transgresión cualitativa.

19 Ibid., p. 24 
Teniendo en cuenta las relaciones sociales de poder de hoy, esto significa, como dice Adorno, que "la única concreción real de la emancipación" consiste "en que las personas que creen necesario caminar en ese sentido influyan del modo más enérgico para que la educación sea una educación para la contradicción y la resistencia" ${ }^{20}$.

${ }^{20}$ Adorno, T. W., Educación para la emancipación. Conferencias y conversaciones con Hellmut Becker (1959-1969), edición de Gerd Kadelbach y traducción de Jacobo Muńoz, Madrid, Ediciones Morata, 1998, p. 125. 


\section{RefERenCias Bibliográficas}

Adorno, Theodor W., Critica de la cultura y sociedad I: Prismas. Sin imagen directriz [ = Obra completa, 10/1, edición de Rolf Tiedemann, traducción de Jorge Navarro Pérez], Madrid, Akal, 2008.

- Dialéctica de la ilustración. Fragmentos filosóficos [= Obra completa, 3, edición de Rolf Tiedemann, traducción de Joaquín Chamorro Mielke], Madrid, Akal, 2007.

- Dialéctica negativa / La jerga de la autenticidad [= Obra completa, 6, edición de Rolf Tiedemann, traducción de Alfredo Brotons Muñoz], Madrid, Akal, 2005.

- Educación para la emancipación. Conferencias y conversaciones con Helmut Becker (1959-1969), edición de Gerd Kadelbach y traducción de Jacobo Muñoz, Madrid, Ediciones Morata, 1998.

- Escritos sociológicos $I$ [ = Obra completa, 8, edición de Rolf Tiedemann, traducción de Agustín González Ruiz], Madrid, Akal, 2004.

Bernhard, Armin, Biopiraterie in der Bildung. Einsprüche gegen die vorherrschende Bildungspolitik, Hannover, Offizin, 2010.

CCE (Comisión de las Comunidades Europeas), Memorándum sobre el aprendizaje permanente, Bruselas, 2000. Documento online disponible en: http://www.oei. es/historicoleduytrabajo2/Memoaprenpermanente.pdf (consultado el 10.06.2018)

CE (Comunidades Europeas), El Marco Europeo de Cualificaciones para el aprendizaje permanente (EQF-MEC), Luxemburgo, Oficina de Publicaciones Oficiales de las Comunidades Europeas, 2009. Documento online disponible en: https:/lec.europa.eu/ploteus/sites/eac-eqfffiles/broch_es.pdf(consultado el 10.06.2018)

Dammer, Karl-Heinz, Vermessene Bildungsforschung. Wissenschaftshistorische Hintergründe zu einem neoliberalen Herrschaftsinstrument, Baltmannsweiler, Schneider Hohengehren, 2015.

Hirsch, Joachim, Materialistische Staatstheorie. Transformationsprozesse des kapitalistischen Staatensystems, Hamburg, VSA, 2005.

Mertens, Dieter, "Schlüsselqualifikationen. Thesen zur Schulung für eine moderne Gesellschaft", en Lange, Elmar y Büschges, Günter (eds.), Aspekte der Berufswahl in der modernen Gesellschaft, Frankfurt a. M., Aspekte-Verlag, pp. 403-429. 
OCDE (Organización para la cooperación y el desarrollo económicos), Conocimientos y aptitudes para la vida. Primeros resultados del programa internacional de evaluación de estudiantes (PISA) 2000 de la OCDE. México, Santillana 2001. Documento online disponible en: http://www.oecd-ilibrary.org/education/conocimientos-y-aptitudes-para-la-vida_9789264065949-es (consultado el 10.06.2018).

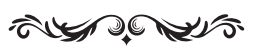

DOI: http://doi.org/10.15366/bp2019.21.005

Bajo Palabra. II Época. No21. Pgs: 97-108 